\title{
Spin-induced deformations and tests of binary black hole nature using third-generation detectors
}

\author{
N. V. Krishnendu, ${ }^{1, *}$ Chandra Kant Mishra, ${ }^{2, \dagger}$ and K. G. Arun ${ }^{1, *}$ \\ ${ }^{1}$ Chennai Mathematical Institute, Siruseri, 603103, India. \\ ${ }^{2}$ Indian Institute of Technology Madras, Chennai, 600036, India.
}

(Dated: March 12, 2019)

\begin{abstract}
In a recent letter [N. V. Krishnendu et al., Phys. Rev. Lett. 119, 091101 (2017)] we explored the possibility of probing the binary black hole nature of coalescing compact binaries, by measuring their spin-induced multipole moments, observed in advanced LIGO detectors. Coefficients characterizing the spin-induced multipole moments of Kerr black holes are predicted by the "no-hair" conjecture and appear in the gravitational waveforms through quadratic and higher order spin interactions and hence can be directly measured from gravitational wave observations. By employing a non-precessing post-Newtonian (PN) waveform model, we assess the capabilities of the third-generation gravitational wave interferometers such as Cosmic Explorer and Einstein Telescope in carrying out such measurements and use them to test the binary black hole nature of observed binaries. In this paper, we extend the investigations of [N. V. Krishnendu et al., Phys. Rev. Lett. 119, 091101 (2017)], limited to measuring the binary's spin-induced quadrupole moment using their observation in second generation detectors, by proposing to measure (a) spin-induced quadrupole effects using third generation detectors, (b) simultaneous measurements of spin-induced quadrupole and octupole effects, again in the context of the third-generation detectors. We study the accuracy of these measurements as a function of total mass, mass ratio, spin magnitudes, and spin alignments. Further, we consider two different binary black hole populations, as proxies of the population that will be observed by the third generation detectors, and obtain the resulting distribution of the spin-induced quadrupole coefficient. This helps us assess how common are those cases where this test would provide very stringent constraints on the black hole nature. These error bars provide us upper limits on the values of the coefficients that characterize the spin-induced multipoles. We find that, using third-generation detectors the symmetric combination of coefficients associated with the spin-induced quadrupole moment of each binary component may be constrained to a value $\leq 1.1$ while a similar combination of coefficients for spin-induced octupole moment may be constrained to $\leq 2$, where both combinations take the value of 1 for a binary black hole system. These estimates suggest that third-generation detectors can accurately constrain the first four multipole moments of the compact objects (mass, spin, quadrupole, and octupole) facilitating a thorough probe of their black hole nature.
\end{abstract}

\section{INTRODUCTION}

Recent detections of binary black hole $(\mathrm{BBH})$ mergers by Laser Interferometric Gravitational wave Observatory (LIGO) and VIRGO gravitational wave observatory have confirmed the existence of binary black holes in nature and that they merge under the effect of gravitational wave $(\mathrm{GW})$ radiation reaction [1-7]. Several tests of general relativity [8-19] were performed using these signals leading to the first ever bounds on potential deviation from the theory in the strong-field regime of gravity $[3-5,20,21]$. These tests make use of the fact that the dynamics of the compact binary, and hence the gravitational waveform could be different in an alternative theory of gravity. Hence the observation of binary black holes can lead to constraints on possible departures from general relativity.

The binary black hole dynamics consists of three major phases inspiral, merger and ringdown. One can model the inspiral phase using post-Newtonian formalism[22] whereas numerical relativity simulations are needed to model the merger regime [23]. In order to study the ringdown part of the dynamics, one may use black hole perturbation theory techniques [24]. While the observations to date are consistent with this binary black hole dynamics, there may still be room for explaining these observed mergers as due to mergers of some exotic com-

\footnotetext{
*Electronic address: krishnendu@ @ cmi.ac.in

${ }^{\dagger}$ Electronic address: ckm@iitm.ac.in

‡Electronic address: kgarun@cmi.ac.in
}

pact objects [25]. These exotic compact objects could mimic the properties of the black holes up to the accuracy with which we are currently able to extract the signal and its parameters. As the gravitational wave interferometers become more and more sensitive, our parameter estimation accuracies should improve dramatically enabling a thorough probe of the nature of these compact objects. Proposed third-generation groundbased detectors such as Einstein Telescope (ET-D) [26] and Cosmic Explorer (CE) [27-29] hence have the strong potential to probe the nature of compact binaries which motivates this work.

Leading candidates for these black hole mimickers include gravastars [30], boson stars [31] and firewalls [32]. Modeling mergers of these exotic objects is a hard problem and their direct deployment for data analysis is not likely to happen in the near future. So a more pragmatic approach would be to devise tests which are generic and model independent and are based on our solid understanding of the binary black hole dynamics. An efficient method to probe the presence of exotic compact objects is to understand the possible ways in which such objects can correct for the properties of black holes which can be detected or ruled out by introducing appropriate free parameters in the gravitational waveform. These tests are often referred to as "null tests" as the free parameters are zero for binary black holes. In order to develop such model independent null tests of black hole mimickers, it is important to identify those properties which are unique to black holes and trace their imprints on the gravitational waveform so that we can measure them from observations.

One of the characteristic properties of black holes in the 
general theory of relativity is related to the "no-hair" conjecture, which says that all the multipole moments of a Kerr black hole are completely specified by its mass and spin. This means that, it is always possible to relate the $\ell^{\text {th }}$ multipole of the Kerr black hole to the mass $(M)$ and the dimensionless spin parameter $\left(\chi=S / M^{2}\right)$ as, $M_{\ell}+\mathrm{i} \mathrm{S}_{\ell}=M^{\ell+1}(\mathrm{i} \chi)^{\ell}[33-38]$. Here $M_{\ell}$ and $S_{\ell}$ are the mass- and the current-type multipole moments, respectively. This property leads to several observational predictions unique to a black hole which are built-in to the gravitational waveform facilitating tests of black hole nature, some of which are discussed below.

\section{A. Tests of binary black hole nature using gravitational waves}

The fact that a black hole cannot be tidally deformed, leads to a vanishing tidal Love number [39, 40]. Using a gravitational wave phasing formula which contains the tidal Love numbers [41, 42], one can directly measure these parameters from observations which in turn can be used to constrain the nature of the compact object constituting the binary system [43-45]. Measurement of tidal deformability parameter from gravitational wave observations for various neutron star models is also studied in different contexts [41]. Recently, Cardoso et al. [43] have calculated the tidal deformability parameters of non-black hole compact objects (including boson stars, gravastars, wormholes, and other toy models for quantum corrections at the horizon scale) and have studied the detectability of such parameters using advanced gravitational wave detectors. In Ref. [44], authors studied the distinguishability of boson star systems from black holes and neutron stars by measuring the tidal deformability parameter. A rigorous formulation of this test using Bayesian inference [45] has brought the idea closer to be implemented on detected gravitational wave events.

Another way to test the black hole nature is by using the quasi-normal modes [46] of the perturbed black hole formed by the merger $[8,47,48,48]$. For a Kerr black hole, all the quasi-normal modes are characterized by the mass and spin of the black hole according to the "no-hair" conjecture. Though the waveform models for exotic compact objects are less developed, there have been various attempts to calculate the quasi normal modes of boson stars [49-51] and gravastars [52-54]. These can be used to discern boson stars and gravastars from black holes.

Measurement of the so called tidal heating parameter can also be used as a tool to test the black hole nature. Consider a black hole event horizon surrounded by external gravitating objects. The rotational energy of this black hole may dissipate gravitationally due to the tidal disruption of exterior matter [55]. The loss of energy and angular momentum of a Kerr black hole near the horizon can lead to non-zero values of the tidal heating parameter. The measured value of the tidal heating parameter will be zero for any system without an event horizon. The tidal heating effect shows up in the gravitational wave phasing $[56,57]$ which helps us to measure this effect from observations [58] and thereby test the black hole nature of the compact object.

It has been found that the multipole moment structure of a central compact object can be extracted from the dynamics of a less massive object orbiting it [59-61]. Reference [62] introduced the "bumpy black holes" as a model of space-times which deviate from that of Kerr black holes. Bumpy black holes and their astrophysical importance is extensively studied in [63].

Recently, Ghosh et. al proposed a method [19, 64] to study the consistency of the inspiral-merger-ringdown dynamics of a binary black hole system to the one predicted by general relativity. The idea here is to infer the mass and spin parameters of the merger remnant from the post-merger part of the gravitational wave signal and ask if this is consistent with the same as inferred from the inspiral part of the gravitational wave signal (using the numerical fitting formula given in [65]). This method allows one to quantify how close the observed high mass compact binary mergers are to the mergers of binary black holes in general relativity $[1,3]$.

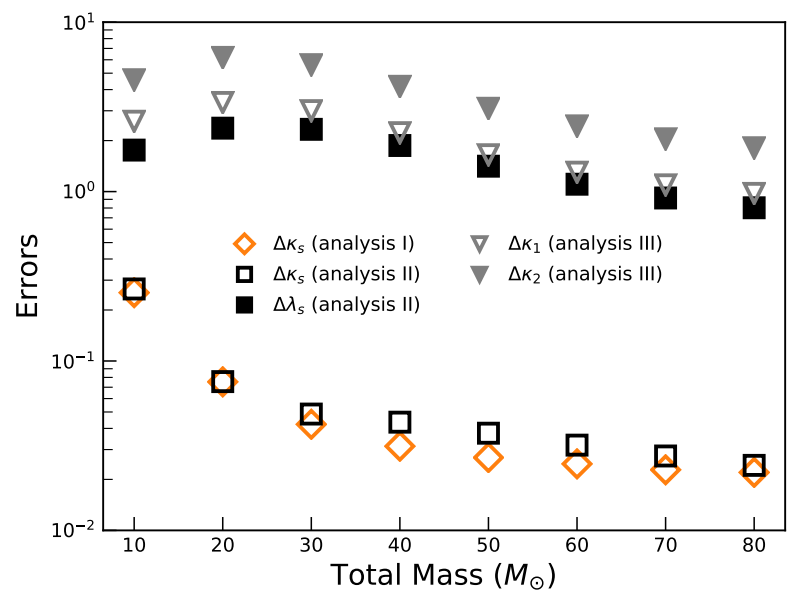

FIG. 1: Figure displays variation of $1-\sigma$ errors in the measurement of parameters characterizing spin-induced multipole moments as a function of the total mass of the binary for the three different analyses. Analysis I represents the case where $\kappa_{s}=\left(\kappa_{1}+\kappa_{2}\right) / 2$ is treated as an independent parameter (here $\kappa_{1,2}$ are parameters characterizing the spin-induced quadrupole moment of each binary component) while the antisymmetric combination of $\kappa_{1}$ and $\kappa_{2}$ as well as the symmetric and antisymmetric combination of parameters characterizing the spininduced octupole moment, $\left(\lambda_{1}, \lambda_{2}\right)$, are set to their binary black hole values of $(0,1,0)$, respectively. In Analysis II, both $\kappa_{s}$ and $\lambda_{s}=$ $\left(\lambda_{1}+\lambda_{2}\right) / 2$ are measured simultaneously while the antisymmetric combination $\kappa_{a}=\left(\kappa_{1}-\kappa_{2}\right) / 2$ and $\lambda_{a}=\left(\lambda_{1}-\lambda_{2}\right) / 2$ are set to their binary black hole values of 0. Finally in Analysis III, we obtain errors on $\kappa_{1}$ and $\kappa_{2}$ while keeping $\lambda_{1}$ and $\lambda_{2}$ to their $\mathrm{BH}$ values of 1 . The binary is assumed to be at a distance of $400 \mathrm{Mpc}$ and is optimally oriented. The binary's mass-ratio is 1.2 and posses spins of 0.9 and 0.8 respectively for heavier and lighter components, respectively.

\section{B. Current work}

Recently we proposed a new method to test the binary black hole nature of coalescing compact binary systems observable by ground-based and space-based gravitational wave interferometers [66]. The method relies on measuring the spin-induced quadrupole moments of the binary constituents, which appear explicitly in the gravitational waveforms. For instance, the spin-induced quadrupole moment is given by $M_{2}=-\kappa \chi^{2} M^{3}$ 

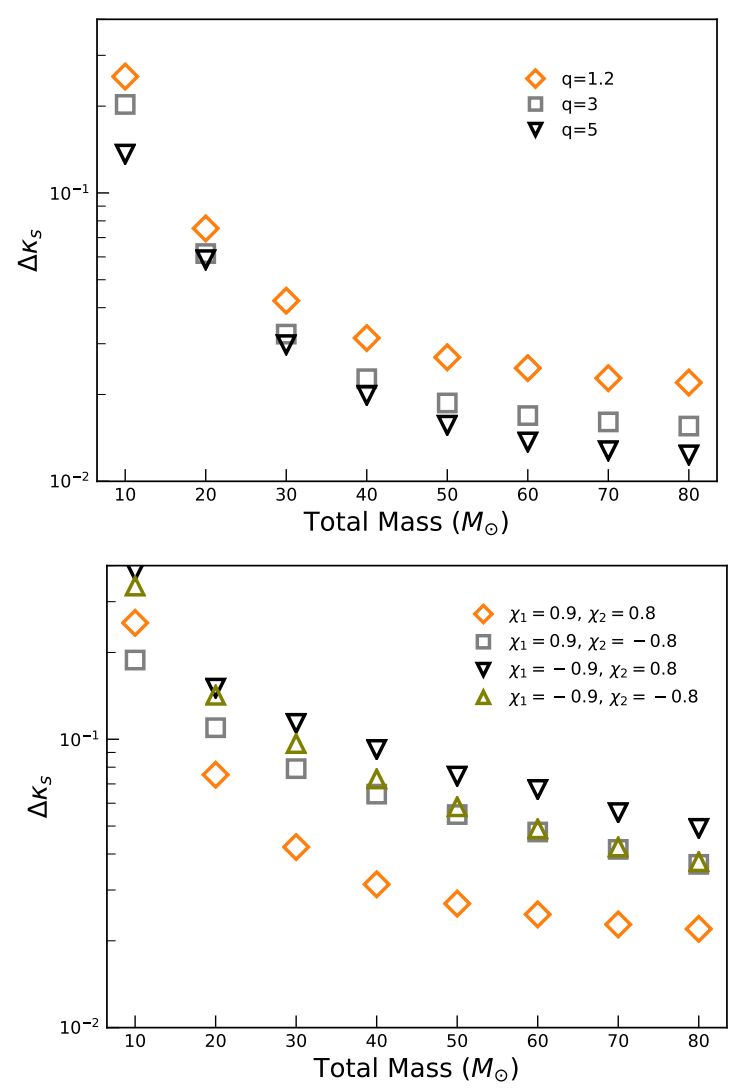

FIG. 2: Figure displays variation of $1-\sigma$ errors on $\kappa_{s}=\left(\kappa_{1}+\kappa_{2}\right) / 2$ (where $\kappa_{1,2}$ are parameters characterizing the spin-induced quadrupole moment of each binary component) as a function of the binary's total mass for three representative mass-ratio cases with fixed component spins $\left(\chi_{1}, \chi_{2}\right)$ of $(0.9,0.8)$ (top panel) and four representative spin configurations with fixed mass-ratio $(\mathrm{q})$ of 1.2 (bottom panel).

where $M$ and $\chi$ are the mass and dimensionless spin parameter of the black hole and the coefficient $\kappa$, which is a measure of the spin-induced quadrupole moment, is unity for Kerr black holes, whereas it can take values roughly between $\sim 2-14$ for neutron stars [67-69] and between $\sim 10-150$ for boson stars [70]. Hence an accurate and independent measurement of this coefficient for each of the binary constituents can tell us if they are indeed black holes [66]. For this purpose, we employed the post-Newtonian (PN) waveforms for spinning compact binaries which are explicitly parametrized in terms of these coefficients (see Sec. II for more details).

It was argued in Ref. [66] that it would not be possible to accurately measure the deformability coefficients associated with each binary constituents $\left(\kappa_{1}, \kappa_{2}\right)$ simultaneously due to the inherent degeneracies between them. However, the symmetric combination of the two, $\kappa_{s}=\left(\kappa_{1}+\kappa_{2}\right) / 2$, can be measured accurately assuming the anti-symmetric combination is zero (which would mean that we work with the condition $\kappa_{1}=\kappa_{2}$ ). Since $\kappa_{1}=\kappa_{2}=1$ for a Kerr black hole (and hence $\kappa_{s}=1$ for a binary black hole), an accurate measurement of $\kappa_{s}$ is an excellent test of the binary black hole nature of the observed compact binary. If the binary system comprises of exotic compact objects, the measurement of the symmetric combination $\kappa_{S}$ should be sensitive to such a deviation from binary black hole nature even if $\kappa_{1} \neq \kappa_{2}$. However, a further analysis, where both $\kappa_{1}$ and $\kappa_{2}$ are simultaneously measured, will be necessary to further understand the composition of the binary and detailed nature of the binary constituents. This possibility is further discussed in Sec. IV C. The error bars associated with the measurement provides the upper limit on the value of $\kappa_{s}$ allowed by the data for black hole mimicker models. These bounds, therefore, can be mapped on the parameter space of various black hole mimicker models. A statistically significant detection of $\kappa_{s} \neq 1$ could be an indication of the presence of exotic physics in play and may be followed up.

In the present work, we extend the idea of [66] in three ways by utilizing the enhanced sensitivity of third-generation detectors $[26,71]$. Firstly, we estimate the errors on $\kappa_{s}$ assuming a third-generation noise sensitivity and find that the enhanced sensitivity of third-generation detectors over secondgeneration detectors improve the $\kappa_{s}$ estimates, roughly, by an order of magnitude (see Fig. 3). Secondly, we investigate the ability of third-generation detectors to simultaneously measure $\kappa_{s}$ and $\lambda_{s}$ (symmetric combination of coefficients associated with spin-induced octupole of each binary component $\left.\left(\lambda_{1}, \lambda_{2}\right)\right)$ while we set the anti-symmetric combinations of each pair of coefficients, $\left(\kappa_{1}, \kappa_{2}\right)$ and $\left(\lambda_{1}, \lambda_{2}\right)$ to zero. This would allow simultaneous measurement of the mass, spin, quadrupole and octupole moments of the source thereby permitting consistency tests between them as tests of the binary black hole nature. Thirdly, we obtain the projected bounds on $\kappa_{1}$ and $\kappa_{2}$ simultaneously using third-generation detectors (keeping the octupole moment coefficients to their BH values). These bounds can straightforwardly be mapped to the black hole nature of the compact object constituting the binary system leading to a much stronger test compared to the one proposed in [66].

A summary of our analysis is shown in Fig. 1, where the projected errors on the measurement of the spin-induced multipole moments for the three scenarios discussed above are shown as a function of total mass for a fixed mass-ratio of 1.2 and dimensionless spin parameters $(0.9,0.8)$. The binary is assumed to be optimally oriented at a luminosity distance of $400 \mathrm{Mpc}$. The projected bounds on the binary black hole nature range from 1 to about 8 for the choice of mass-ratio and spin values depending on the type of test performed. We see in Fig. 1 that $\kappa_{s}$, whether measured alone (Analysis I) or together with $\lambda_{s}$ (Analysis II) is measured with the smallest errors. We also note that the addition of $\lambda_{s}$ to the parameter space does not affect the errors on $\kappa_{S}$ as they are relatively less correlated because of the different PN orders at which they appear unlike $\kappa_{1}$ and $\kappa_{2}$ which are strongly correlated as they occur together in the phasing.

The rest of the paper is organized in the following way. In Sec. II, we review the idea of spin-induced multipole moments of compact binary system within the post-Newtonian (PN) formalism. We will briefly describe the aspects of the Fisher information matrix in Sec. III. Section IV reports the results in detail. We conclude with Sec. V. 

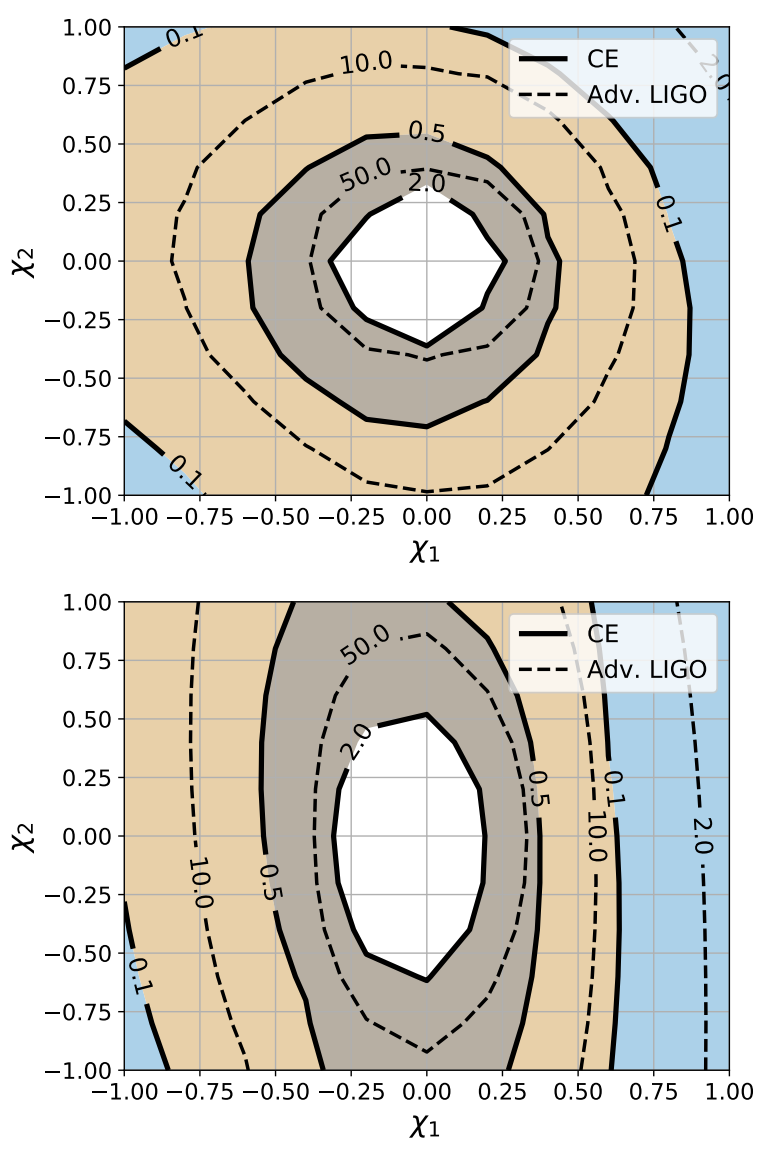

FIG. 3: The errors on $\kappa_{s}$, the symmetric combination of $\kappa_{1}$ and $\kappa_{2}$, in the dimensionless spin parameter plane for the binary system with total mass of $30 M_{\odot}$ and mass-ratios of $q=1.2$ (top panel) and $q=3$ (bottom panel). We assume the binary to be optimally oriented at a luminosity distance of $400 \mathrm{Mpc}$. In both panels, the solid curve corresponds to the errors using Cosmic Explorer noise PSD and the errors using advanced LIGO noise PSD is denoted by dashed contours. As can be seen from the plots, parameter space explored in the $\chi_{1^{-}}$ $\chi_{2}$ plane is much larger for Cosmic Explorer compared to advanced LIGO.

\section{SPIN-INDUCED MULTIPOLE MOMENTS IN THE POST-NEWTONIAN WAVEFORMS}

Evolution of a compact binary system during the inspiral phase is accurately modeled by the post-Newtonian formalism (see [72] for a review). While sufficiently accurate postNewtonian gravitational waveforms (for the purposes of detection and the parameter estimation) from compact binaries with non-spinning constituents in quasi-circular orbits were made available as early as the early 2000s [73-75], higher order spin effects were included through a number of recent investigations [76-86]. For our purposes, we choose to work with a frequency domain waveform where the spins are (anti-) aligned with respect to the orbital angular momentum [86]. The stateof-the-art frequency domain waveform for compact binaries with (anti-) aligned spin components incorporates spin-orbit effects in phasing up to 4PN (leading effect appears at 1.5PN order in the phase), spin-spin effects up to 3PN (starting at $2 \mathrm{PN}$ ) and the leading cubic-spin terms at 3.5PN. Moreover, the amplitude involves spin effects up to $2 \mathrm{PN}$.

The waveform we use for our analyses contain only the leading (second) harmonic (quadrupolar mode) and its PN corrections in the amplitude, while the presence of higher modes in the waveform is neglected, and schematically reads as,

$$
\tilde{h}(f)=\frac{M^{2}}{D_{L}} \sqrt{\frac{5 \pi \eta}{48}} \sum_{n=0}^{4} V_{2}^{n-7 / 2} C_{2}^{(n)} \mathrm{e}^{\mathrm{i}\left(2 \Psi_{\mathrm{SPA}}(f / 2)-\pi / 4\right)},
$$

where $M, \eta$ and $D_{L}$ denote the total mass, symmetric mass-ratio and the luminosity distance to the binary system respectively. Coefficients $C_{2}^{(n)}$ represent the amplitude corrections to the quadrupolar harmonic at (n/2) PN order [82]. The pre-factor $V_{2}$ related to the gravitational wave frequency $(f)$ and the total mass of the binary system as, $V_{2}=(\pi M f)^{1 / 3}$. Here $\Psi_{\mathrm{SPA}}(f)$ represents the phase of the waveform. Each of these $C_{2}^{(n)}$ and the phasing, with explicit dependence on spin-induced quadrupole (through $\kappa_{s}$ and $\kappa_{a}$ ) and octupole (through $\lambda_{s}$ and $\lambda_{a}$ ) moment parameters at respective PN orders are given in supplemental material of [66].

Effect of the leading spin-induced multipole moment (masstype quadrupole, $M_{2}=-M^{3} \chi^{2}$ ) in the phasing of gravitational waves from binary black hole systems was first computed in [87] and contributes to the gravitational wave phase at 2PN order. Here, the symbols $M$ and $\chi$ again represent the mass and dimensionless spin parameter for each binary component while the negative sign (by convention) indicates that the spin induces oblateness to the black hole. Post-Newtonian corrections to this at 3PN order has been computed in [80]. The sub-leading, spin-induced multipole moment (current-type octupole, $S_{3}=$ $-M^{4} \chi^{3}$ ) starts to contribute to the phase at 3.5PN order and was computed in [81]. Notice, the spin dependences of the spin-induced multipole moments here: $M_{2}\left(S_{3}\right)$ have quadratic (cubic) dependences on the spin parameter and first appear in the phasing formula at $2 \mathrm{PN}(3.5 \mathrm{PN})$ order because these are the orders at which quadratic-in-spin (cubic-in-spin) terms start to appear in the gravitational wave phase.

Note that the relations for $M_{2}$ and $S_{3}$ assume that the binary constituents are black holes but can be generalized for a non-BH compact object by introducing coefficients that characterize the degree of deformation. For instance, we can rewrite these relations as : $M_{2}=-\kappa M^{3} \chi^{2}$ and $S_{3}=-\lambda M^{4} \chi^{3}$ where the coefficients $\kappa$ and $\lambda$ take the value unity for Kerr black holes whereas they deviate from unity for other types of compact objects including exotic alternatives to black holes. For example, the values of $\kappa$ and $\lambda$ for neutron stars, depending upon the neutron star equation of state and mass, range between $\sim 2-14$ and $\sim 4-30$, respectively [67-69]. The spin-induced multipole moments of a few exotic compact objects are also computed in the literature: for a particular class of spinning boson star system $\kappa(\lambda)$ can take values between $\sim 10-150(\sim 10-200)$ [70]. Variation of quadrupole and octupole moment parameters in the boson star mass-spin parameter plane is shown respectively in Figs. 4 and 5 of [70]. Similar computations have been done for gravastars, see for instance Refs. [88-90] which discuss spin-induced multipole moments for thin shell gravastar models. If the observed values of spin-induced quadrupole moments are offset from black 


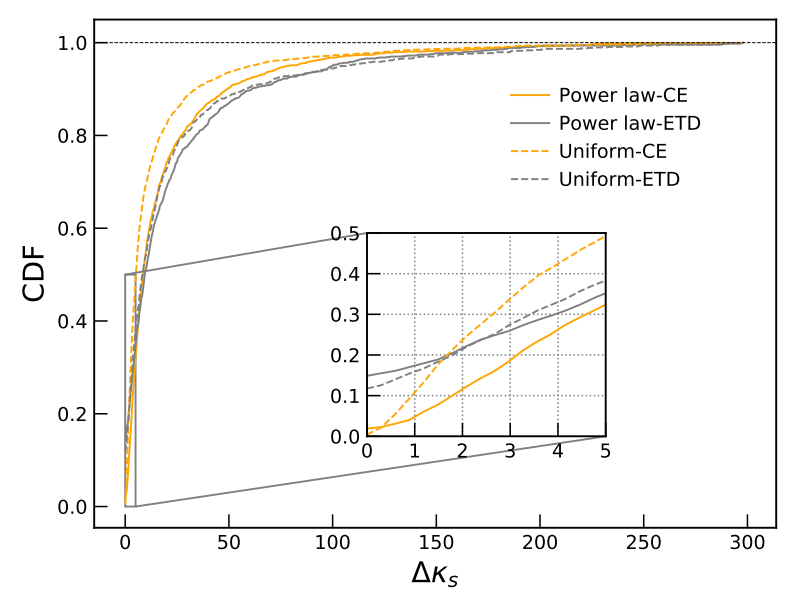

FIG. 4: The cumulative distribution function of errors on $\kappa_{s}$ for two prototypical astrophysical populations of binary black holes corresponding to two different models for the binary's mass distribution. In the first model we assume both component masses to be uniformly distributed between $5 M_{\odot}$ to $20 M_{\odot}$ while the second model assumes the primary mass to follow a power-law distribution with an index $\alpha=2.3[3,6]$ and uniform distribution for the secondary. In both the models the masses are defined with respect to the source frame and the sources are distributed uniformly in the comoving volume up to a redshift of 1 .

hole value, it may be interpreted as an evidence of an exotic compact object. On the other hand, if the posterior distribution for the observed value is found to be peaking at 1 with a width, the corresponding error bars can be translated into an upper bound on the allowed value of the parameter for the particular system. In this work, we compute the projected accuracies on the measurement of the spin-induced multipole moments using the semi-analytical parameter estimation technique of the Fisher information matrix. The necessary details of the scheme and the analysis are presented in the next section.

\section{PARAMETER ESTIMATION USING THE FISHER INFORMATION MATRIX ANALYSIS}

When we have an accurate model for the signal of interest and the expected sensitivity of the detector, Fisher information matrix approach can be used to compute the 1- $\sigma$ error bars on the parameters of the signal [91] assuming the noise in the detector is Gaussian-stationary and the signal-to-noise ratio is high. Here we employ this approach to estimate the possible error bars on parameters associated with spin-induced multipole moment of the compact binary system. A quick review of Fisher information matrix formalism is given here. More details can be found in [91].

A detector output consisting of the gravitational wave signal and the background noise can be written as,

$$
s(t)=h\left(t ; \theta_{i}\right)+n(t)
$$

where $h\left(t ; \theta_{i}\right)$ is the true signal which is buried in the noise $n(t)$ and $\theta_{i}$ represents the set of parameters that characterizes the signal. Due to the presence of noise, the measured parameters $\theta_{i}$ can fluctuate about the true value leading to errors associated with their measurements. Hence measured value of $\theta_{i}=\theta_{i}^{\text {true }} \pm$ $\Delta \theta_{i}$, where $\theta_{i}^{\text {true }}$ is the true value of the parameter and $\Delta \theta_{i}$ is the error associated with the measurement due to noise, give us information about the parameter $\theta_{i}$. From the measurement, we are interested in the probability distribution function for $\theta_{i}$ given the signal $s(t), p\left(\theta_{i} \mid s\right)$. It can be shown that, for Gaussian noise in the limit of high signal-to-noise ratios, the posterior probability takes the form,

$$
p\left(\theta_{i} \mid s\right) \propto \mathrm{e}^{-\frac{1}{2}\left(\Gamma_{j k} \Delta \theta_{j} \Delta \theta_{k}\right)},
$$

where $\Gamma_{i j}$ is called the Fisher information matrix $[92,93]$ defined as follows,

$$
\Gamma_{i j}=2 \int_{f_{\text {lower }}}^{f_{\text {upper }}} d f \frac{\tilde{h}_{i}(f) \tilde{h}_{j}^{*}(f)+\tilde{h}_{j}(f) \tilde{h}_{i}^{*}(f)}{S_{n}(f)},
$$

where $S_{n}(f)$ represents the noise power spectral density (PSD) of the detector and $\tilde{h}_{i} \equiv \partial \tilde{h}\left(f ; \theta_{i}\right) / \partial \theta_{i}$ is evaluated at the true value of the parameter $\theta_{i}=\theta_{i}^{\text {true }}$. Inverse of the Fisher information matrix is called the covariance matrix $\left(\Sigma_{i j}\right)$ and the error $\left(\sigma_{i}\right)$ on each parameter $\theta_{i}$ is given by the square root of the diagonal entries of the covariance matrix. That is,

$$
\sigma_{i}=\sqrt{\Sigma_{i i}}
$$

We choose to terminate the integral of Eq. (3.3) at twice the orbital frequency of the inner most stable circular orbit $\left(f_{\text {ISCO }}\right)$ for a spinning compact binary and use the fits obtained in $[94,95]^{1}$. The lower frequency cut-off in the integral of Eq. (3.3) is fixed by the sensitivity of the detector given by the function $S_{n}(f)$. In this work, we intend to explore the parameter estimation analysis for two different third-generation gravitational wave detector configurations: Cosmic Explorer (CE) [96, 97] and Einstein Telescope (ET-D) [97]. Since the two have comparable sensitivities and we choose one of them (in our case CE noise PSD) for the most part of the paper. However, we compare the performance of CE and ET-D for a few representative cases. The low frequency cut-off for $\mathrm{CE}$ (ET-D) configuration is chosen to be $5 \mathrm{~Hz}(1 \mathrm{~Hz})$ which defines the $f_{\text {low }}$ value we use in the integral given in Eq. (3.3). We also discuss the improvements one expect due to the use of third-generation detector sensitivities over advanced LIGO and choose low frequency cut-off as $20 \mathrm{~Hz}$ for advanced LIGO.

\section{RESULTS AND DISCUSSIONS}

In this section, we present the results of our analyses. We perform the parameter estimation analysis for a set of prototypical (stellar mass) compact binary systems with the assumption that the binaries are optimally oriented and are located at a fiducial distance of $400 \mathrm{Mpc}$. The component spin magnitudes are

\footnotetext{
${ }^{1}$ Here we only consider contributions from the second harmonic as discussed in Sec. II.
} 


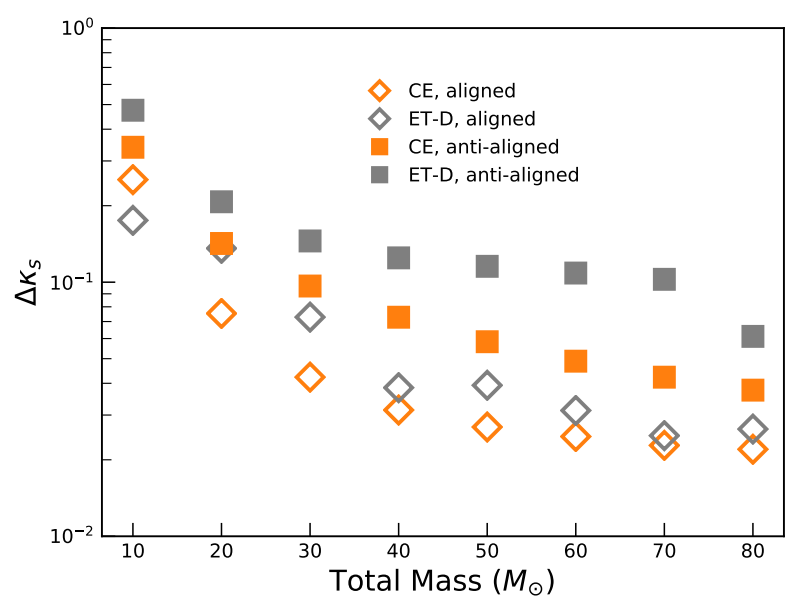

FIG. 5: Errors on the $\kappa_{s}$ as a function of the total mass of the binary system for two representative 3rd generation detectors, Cosmic Explorer (CE noise PSD) and Einstein Telescope (ET-D noise PSD). The binary is assumed to be at a distance of $400 \mathrm{Mpc}$ and is optimally oriented. The binary's mass-ratio is 1.2 and spin magnitudes of 0.9 and 0.8 for heavier and lighter components, respectively. Filled- (empty-) markers represent spin orientations of each component aligned (antialigned) to the orbital angular momentum while squares (diamonds) represent error estimates for Cosmic Explorer (Einstein Telescope, ET-D).

represented by the dimensionless spin parameter, $\chi_{1,2}$, where subscripts 1(2) represents the primary (secondary) binary component. We also follow the convention to assign higher mass and spin values to the primary component. In addition to this, we also obtain a distribution of errors of the spin-induced multipole moment parameters for a simulated population of binary black holes, which act as proxies for the binary black hole population third generation detectors would observe.

As discussed above, we choose to work with the Cosmic Explorer noise PSD as a representative noise sensitivity of a third-generation detector configuration [27-29]. The lower (upper) frequency cut-offs appearing in Eq. (3.3) are chosen to be $5 \mathrm{~Hz}\left(2 \times f_{\mathrm{ISCO}}\right.$ for spinning binary black holes $\left.[94,95]\right)$. These results are compared with the corresponding ones for advanced LIGO and Einstein Telescope for a selected set of binary configurations.

\section{A. Bounds on binary's spin-induced quadrupole moment parameter}

If we assume the two objects in the binary system suffer equal deformation due to their individual spins ( i.e., $\kappa_{1}=\kappa_{2}$ ), the symmetric combination of the coefficient of spin-induced quadrupole moments, $\kappa_{s}$, will be the suitable parameter to constrain the binary black hole nature [66]. Any deviation from the binary black hole value of $\kappa_{s}=1$ can be interpreted as a possible constraint on the binary black hole nature of the compact binary system. The parameter space considered here is the following,

$$
\theta_{i}=\left\{t_{c}, \phi_{c}, M_{c}, \eta, \chi_{1}, \chi_{2}, \kappa_{s}\right\}
$$

where $t_{c}$ and $\phi_{c}$ are the time and phase at coalescence, $M_{c}$ $\left(M_{c}=M \eta^{3 / 5}\right)$ is the chirp mass, $\eta=\frac{m_{1} m_{2}}{\left(m_{1}+m_{2}\right)^{2}}$ is the symmetric mass-ratio, $M=m_{1}+m_{2}$ is the total mass and $m_{1}, m_{2}$ and $\chi_{1}, \chi_{2}$ are the masses and dimensionless spin parameters of the binary constituents. Note that, here $\kappa_{s}$ is the only spininduced parameter that is considered free in the analysis; other combinations, $\left(\kappa_{a}, \lambda_{s}, \lambda_{a}\right)$, are set to their binary black hole values of $\kappa_{a}=0, \lambda_{s}=1$ and $\lambda_{a}=0$.

Figure 2 shows the variation of the errors in the measurement of the parameter $\kappa_{s}$, as a function of the total mass of the binary. These errors also provide us $1-\sigma$ upper bounds on the value of $\kappa_{s}$. Three different set of markers in the top panel plot correspond to three different mass-ratios $(\mathrm{q}=1.2,3,5)$ while the component spins are fixed to the values of $\chi_{1}=0.9$, $\chi_{2}=0.8$. On the other hand, the bottom panel assumes a binary with fixed mass-ratio $(q=1.2)$ and displays the errors for four different spin configurations. Each set of markers in both panels suggest that errors decrease as the binary's mass increases. This is largely due to larger signal-to-noise ratios associated with heavier binaries with fixed mass-ratio and component spins. In addition, the trends displayed in the top panel suggest improved $\kappa_{S}$ estimates for larger mass-ratio cases (though the improvement is very minor) while those in the bottom panel show that the best $\kappa_{s}$ estimates correspond to the case when the two objects have component spins aligned to the orbital angular momentum. The improved $\kappa_{s}$ estimates with respect to the mass ratio may be attributed to the larger number of gravitational wave cycles for asymmetric systems in the detector band. Similarly, as the upper cut-off frequency for aligned spin configuration is larger, leading to larger number of gravitational wave cycles, the error estimates for aligned spin configurations are the best.

Figure 3 explores $\kappa_{s}$ error estimates in component spin parameter space for a binary with total mass of $30 M_{\odot}$ and massratios of 1.2 (top panel) and 3 (bottom panel). Solid (dashed) contours represent errors on $\kappa_{s}$ in the context of CE (advanced LIGO) detector. We can compare the performance of advanced LIGO and CE at those points where their contours intersect. It is obvious from the figure that the typical improvements in the estimation of $\kappa_{s}$ due to CE is by a factor of $\sim 40-50$. This improvement is correlated with the increased signal-to-noise ratio of the sources in the CE band compared to advanced LIGO. It is worth noting that, even though the overall improvement in sensitivity of CE over advanced LIGO is roughly a factor of 10 , due to the larger band width of $\mathrm{CE}$, the signal-to-noise ratios are higher than advanced LIGO roughly by $\sim 40-50$ which explains the overall improvement in the parameter estimation of CE with respect to advanced LIGO.

Another striking feature in Fig. 3 is the shape of the contours in the component spin plane. For nearly equal mass systems ( $q=1.2)$, both advanced LIGO and CE contours are nearly circular, whereas for $q=3$ they are ellipses. This feature may be explained by a close inspection of the structure of the leading order spin-spin dependence in the phasing which is proportional to the $\kappa_{s}$ parameter. The term schematically reads as $\Psi_{\text {spin-spin }} \sim \kappa_{s} \zeta\left(\eta, \chi_{1}, \chi_{2}\right)$, where

$$
\zeta\left(\eta, \chi_{1}, \chi_{2}\right)=\alpha(\eta) \chi_{1}^{2}+\beta(\eta) \chi_{2}^{2} .
$$



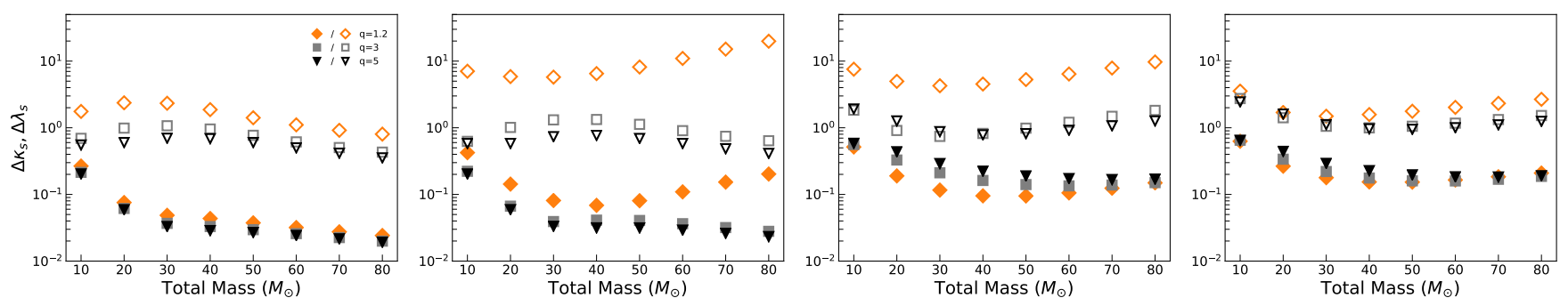

FIG. 6: Figure displays variation of $1-\sigma$ errors on $\kappa_{s}$ (filled markers) and $\lambda_{s}$ (unfilled markers) as a function of the binary's total mass for three representative mass-ratio cases and four representative spin-orientations with fixed component spin magnitudes $\left(\chi_{1}, \chi_{2}\right)$ of $(0.9,0.8)$. The four panels (left to right) represent binaries where spins of the two BHs are aligned, heavier one aligned and the other anti-aligned, heavier one anti-aligned and the other aligned and both the spins are anti-aligned to the orbital angular momentum axis. We assume the binary to be optimally oriented at a luminosity distance of $400 \mathrm{Mpc}$.

Here

$$
\alpha(\eta)=(1+\sqrt{1-4 \eta}-2 \eta)
$$

and

$$
\beta(\eta)=(1-\sqrt{1-4 \eta}-2 \eta)
$$

The derivative of the waveform with respect to $\kappa_{s}$ now will scale as $\sim \zeta$ and the corresponding Fisher information matrix element will scale as $\Gamma_{\kappa_{s} \kappa_{s}} \sim \zeta^{2}$. Intuitively, as the error on $\kappa_{s}$ is proportional to the square root of the inverse of the Fisher matrix, we find $\Delta \kappa_{s} \sim \zeta^{-1}$. Now the contours of constant errors in the component spin plane have the form,

$$
\chi_{1}^{2}\left(\alpha \Delta \kappa_{s}\right)+\chi_{2}^{2}\left(\beta \Delta \kappa_{s}\right)=1 .
$$

It is now obvious that for equal mass systems for which $\alpha=\beta$, the contours of constant errors should be circles whereas for unequal mass systems the contours will be ellipses. From Eq. (4.3), as $\frac{1}{\sqrt{\alpha}} \leq \frac{1}{\sqrt{\beta}}$, these ellipses will have their semimajor axis along $\chi_{2}$ direction as seen in the bottom panel of Fig. 3. Though this scaling completely neglects the correlation of $\kappa_{s}$ with other parameters, this does give us a qualitative picture about the shape and orientation of the contours.

We also explore the performance of the proposed test on an astrophysical population of binary black holes that the thirdgeneration detectors may see by simulating two populations of binary black holes which correspond to different models for the component mass distribution. In the first model, we distribute the source frame component masses $m_{1,2}$ (here $m_{1}>m_{2}$ ) uniformly between $5 M_{\odot}$ and $20 M_{\odot}$. The second model assumes a power-law distribution with an index $\alpha=2.3[3,6]$ for the primary and uniform distribution for the secondary, again, with masses between $5 M_{\odot}$ to $20 M_{\odot}$. For both these cases, we distribute sources with constant comoving number density up to a redshift of $z=1$. The source locations and orientations are uniform on the sky and the polarization spheres, respectively. In order to account for the cosmological redshift on the gravitational signal we rescale the source frame masses $\left(m_{s}\right)$ to redshifted masses $\left(m_{d}\right)$ as, $m_{d}=m_{s}(1+z)$ in the gravitational wave signal while performing parameter estimation using Fisher matrix. This means that the maximum and min- imum component masses in the detector frame will be $5 M_{\odot}$ and $40 M_{\odot}$, respectively. We randomly draw 2000 sources from this population and perform the Fisher analysis to obtain the errors on various parameters including $\kappa_{s}$. Figure 4 shows the resulting distribution of errors on $\kappa_{s}$ for the two populations described above using Einstein Telescope and Cosmic Explorer. As can be seen in the inset of Fig. 4, use of the uniform over power-law distribution leads to nearly $20 \%$ increase in the population of binaries observed with $\Delta \kappa_{s} \leq 5$ for Cosmic Explorer whereas the errors we get using Einstein Telescope are largely independent of the mass distribution. Furthermore, we find that errors on $\kappa_{s}$ are less than 10 for $52 \%(68 \%)$ of the sources for the power-law (uniform) distribution model if we assume CE sensitivity. The numbers change to $41 \%$ and $45 \%$ respectively for power-law and uniform distributions when we consider Einstein Telescope. These trends can be understood as follows: the mass ratio distribution with primary's mass distributed using the power-law leads to fewer sources with larger mass ratios compared to the case where we assume uniform distribution for component masses. In addition, the proposed test is more effective when the mass ratios are higher (see Fig. 2). These two factors improve the overall performance of the test for the uniform mass distribution as can be seen in Fig. 4.

Finally, Fig. 5 compares $\kappa_{s}$ estimates obtained using two different third-generation detector configurations, Cosmic Explorer (CE) and Einstein Telescope (ET-D). In this case, errors on $\kappa_{s}$ as a function of total mass for a fixed mass-ratio of 1.2 is shown. We consider two spin orientations here, both the black holes aligned and both the black holes anti-aligned to the orbital angular momentum axis. As we expect, the performance of CE and ET-D detectors are comparable. However, the Cosmic Explorer error estimates are marginally better than ET-D for all cases except at low masses when component spins are aligned with respect to the orbital angular momentum. This should be a reflection of the improved low frequency sensitivity of ET-D at frequencies less than $5 \mathrm{~Hz}$.

\section{B. Simultaneous bounds on binary's spin-induced quadrupole and octupole moment parameters}

Below we discuss the measurability of both the quadrupolar and octupolar spin-induced deformations due to individual 
BH spins, simultaneously. This time we intend to measure a symmetric combination of coefficients characterizing the spin-induced octupole moment of the compact binary system: $\lambda_{s}=\left(\lambda_{1}+\lambda_{2}\right) / 2$ along with the parameter $\kappa_{s}$. Again the antisymmetric combinations $\kappa_{a}$ and $\lambda_{a}$ are set to their binary black hole value of zero. Formally, simultaneous bounds on $\kappa_{s}$ and $\lambda_{s}$ are more stringent than the $\kappa_{s}$ alone as we are sensitive to two of the leading spin-induced multipoles instead of one. The parameter space considered for this analysis is,

$$
\theta_{i}=\left\{t_{c}, \phi_{c}, M_{c}, \eta, \chi_{1}, \chi_{2}, \kappa_{s}, \lambda_{s}\right\}
$$

where all the parameters have their usual meaning.

Figure 6 shows variations in estimating bounds on $\kappa_{s}$ (filled markers) and $\lambda_{s}$ (unfilled markers) as a function of the total mass of the binary for three different mass-ratios $(q=1.2,3,5)$ and for fixed spin magnitudes of 0.9 and 0.8. Spin orientations chosen are those where both the black hole spins aligned, heavier black hole spin aligned and other anti-aligned, heavier black hole spin anti-aligned other aligned and both the spins anti-aligned to the orbital angular momentum axis, respectively from left to right of Fig. 6.

As discussed in Sec. II, spin-induced octupole moment terms start to appear at 3.5 PN order in the PN phasing formula, while the leading spin-induced quadrupole moment contributes at the 2PN order and hence is a dominant effect in the PN dynamics. Hence, among $\kappa_{s}$ and $\lambda_{s}$ the better constrained parameter is always $\kappa_{s}$. From Fig. 6, it is clear that the $\kappa_{s}$ errors are almost an order of magnitude better estimated compared to $\lambda_{s}$ errors and it is evident from the same figure that, for most of the parameter space, the errors on $\kappa_{s}$ is unaffected due to the inclusion of $\lambda_{s}$ in the problem.

Figure 6 also shows that the bounds on both $\kappa_{s}$ and $\lambda_{s}$ are tightly constrained for cases where the spin of the heavier black hole aligned to the orbital angular momentum axis and if the binary is more asymmetric. When both spins are aligned with respect to the orbital angular momentum, the effect of mass-ratio is marginal (similar to the case presented in Sec. IV A where only $\kappa_{s}$ is measured). On the other hand, having the lighter component anti-aligned with respect to the orbital angular momentum vector only marginally affects the measurements, with the most affected cases being the symmetric systems. We also note that the trends are not clear when we deal with cases where heavier or both components are anti-aligned. In any case, we do not expect the best results when heavier or both components are anti-aligned.

The effect of spin magnitudes on the error estimates for simultaneous $\kappa_{s}$ (top panel) and $\lambda_{s}$ (bottom panel) measurements are shown in Fig. 7. We choose a total mass of $30 M_{\odot}$ and mass ratios of $q=1.2$ (solid contours) and $q=3$ (dotted contours) for this case. Broadly the features seen here resemble those of Fig. 3 where only $\kappa_{s}$ was estimated. For nearly equal mass systems, we see that the contours are less circular when $\lambda_{s}$ is included as a parameter. This may be due to the degeneracies brought in by the estimation of $\lambda_{s}$. Regarding the contours of constant error on $\lambda_{s}$ (bottom panel of Fig. 7), following a line of argument similar to the one in Sec. IV A, it can be shown that the equations of the contours should schematically read as $a \chi_{1}^{3}+b \chi_{2}^{3}=1$, where $a, b$ are functions of mass-ratio which decide shape and orientation of the contours.
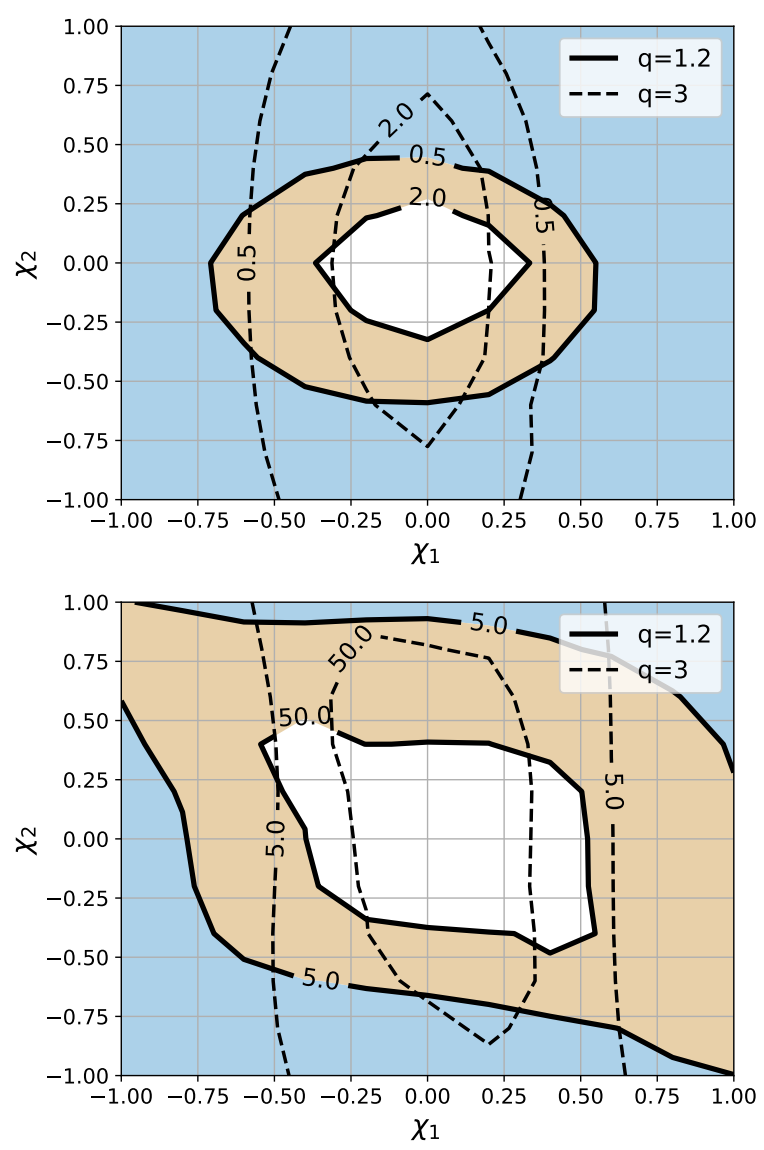

FIG. 7: Errors on spin-induced quadrupole and octupole moment parameters of the binary- $\kappa_{s}$ (top panel) and $\lambda_{s}$ (bottom panel) in the $\chi_{1}-\chi_{2}$ plane for a binary system with total mass $30 M_{\odot}$. Solid contours represent mass-ratio of 1.2 and dashed ones represent mass-ratio 3. Binary system is assumed to be optimally oriented at a luminosity distance of $400 \mathrm{Mpc}$.

We performed an analysis, similar to the one reported in Sec. IV A, where we simulated two populations of binary black holes following a uniform and power-law distributions for the mass of the binary's primary (heavier) component in the source frame, keeping the secondary component mass to be uniformly distributed such that the total mass is less than or equal to $40 M_{\odot}$. We then compute the distribution of the bounds expected from the resulting population. Our analysis show that when $\kappa_{s}$ and $\lambda_{s}$ are measured simultaneously, errors on $\lambda_{s}$ are less than 10 for about $\sim 6 \%(4 \%)$ sources when we use power-law (uniform) distribution on component masses for Cosmic Explorer. As observed earlier $\kappa_{s}$ estimates are marginally affected compared to the case when $\kappa_{s}$ alone is measured. We find that for nearly $42 \%(51 \%)$ sources $\Delta \kappa_{s} \leq 10$ with power-law (uniform) distribution when measured along with $\lambda_{s}$. Again the error distribution for $\kappa_{s}$ is similar to those in Fig. 4. 

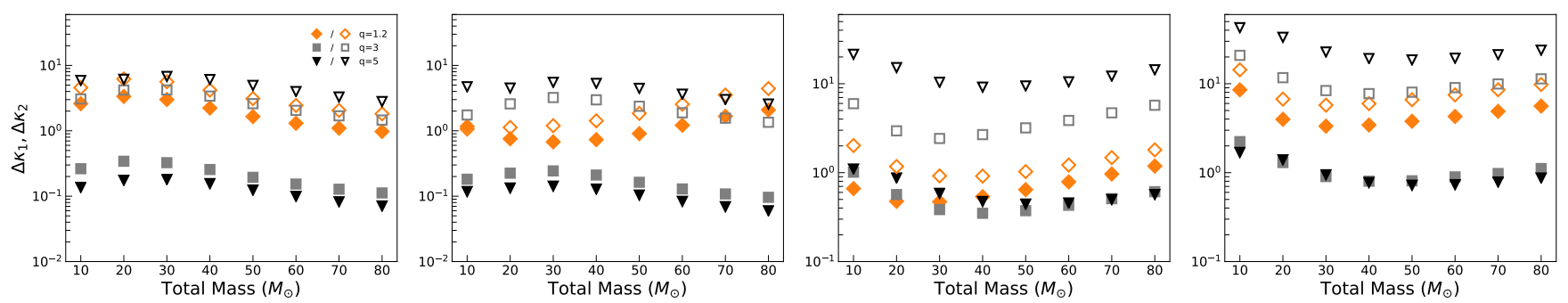

FIG. 8: Figure displays variation of $1-\sigma$ errors on $\kappa_{1}$ (filled markers) and $\kappa_{2}$ (unfilled markers) as a function of the total mass of the binary system for three representative mass-ratio cases and four representative spin-orientations with fixed component spin magnitudes $\left(\chi_{1}, \chi_{2}\right)$ of $(0.9,0.8)$. The four panels (left to right) represent binaries where spins of the two BH are aligned, heavier one aligned and the other anti-aligned, heavier one anti-aligned and the other aligned and both the spins are anti-aligned to the orbital angular momentum axis. Also, note the (up-scaled) y-axes in last two panels.

\section{Bounding the black hole nature of the compact binary constituents}

In this section, we turn to our third and final analysis item - measuring both $\kappa_{1}, \kappa_{2}$ that characterize the spin-induced quadrupole moment coefficients of the binary components. Recall that simultaneous measurement of both $\kappa_{1}, \kappa_{2}$ provides a much stronger test compared to earlier cases where we assumed the spin-induced multipole coefficients to be the same for both the components of the binary $\left(\kappa_{1}=\kappa_{2}, \lambda_{1}=\lambda_{2}\right)$. The parameter space explored in this case is as follows,

$$
\theta_{i}=\left\{t_{c}, \phi_{c}, M_{c}, \eta, \chi_{1}, \chi_{2}, \kappa_{1}, \kappa_{2}\right\},
$$

where the parameters have usual meaning.

Figure 8 shows variations in errors on $\kappa_{1}$ (filled markers) and $\kappa_{2}$ (empty markers) as a function of total mass of the binary for three different mass ratios $(q=1.2,3,5)$ and four different spin configurations (each with fixed spin magnitudes of 0.9 and 0.8 for the heavier and lighter component, respectively). Here again, the spin orientations chosen are those where both the black hole spins aligned, heavier component aligned and other anti-aligned, heavier component anti-aligned other aligned and both the spins anti-aligned to the orbital angular momentum axis, respectively from left to right of Fig. 8. One of the first things we observe is that estimates of $\kappa_{1}$ (which characterizes spin-induced deformations of the heavier $\mathrm{BH}$ ) is consistently better than those of $\kappa_{2}$ (which characterizes spininduced deformations of the lighter $\mathrm{BH}$ ) for all mass-ratios and spin configurations. We also note that $\kappa_{1}$ is measured with smaller errors for systems which are more asymmetric and if the heavier BH is aligned with the orbital angular momentum axis.

These trends can be understood from the leading order spininduced quadrupole moment term in the gravitational wave phasing formula which is proportional to $\kappa_{1} \alpha(\eta) \chi_{1}^{2}+\kappa_{2} \beta(\eta) \chi_{2}^{2}$ as we explained in Sec. IV A. As $\alpha(\eta) \geq \beta(\eta)$ and as we assign larger spin values to the more massive component, for any given spin configuration the pre-factor of $\kappa_{1}$ is always higher than that of $\kappa_{2}$. This explains why $\kappa_{1}$ estimates are better than $\kappa_{2}$. Further, as $\alpha(\eta)$ increases with mass-ratio, the error on $\kappa_{1}$ improves with mass asymmetry. Similarly, the errors on $\kappa_{2}$ worsens with increase in the mass-ratio, since $\beta(\eta)$ is a decreasing function of the mass-ratio.

\section{CONCLUSION}

In three different sets of numerical experiments discussed above, we find that improved sensitivities of third-generation detectors (Cosmic Explorer or Einstein Telescope) over the current advanced LIGO detectors not only allow us to significantly constrain the leading spin-induced effects in gravitational waveforms but also enable us to explore a much wider mass and spin parameter space (Sec. IV A). Assuming an astrophysical population of binary black holes, we show that the errors on spin-induced quadrupole moment parameter is $\leq 5$ for $30 \%$ of the total population if we assume the primary component masses follow the power-law distribution when CE configuration is used. This fraction is roughly $20 \%$ larger if the component masses are uniformly distributed while the errors with Einstein Telescope are largely independent of the mass distribution as can be seen from Fig. 4. As expected, estimated bounds using the two third-generation detectors (Cosmic Explorer or Einstein Telescope) are comparable with a slight favor towards Cosmic Explorer configuration for high mass systems whereas the low mass, aligned spins systems benefit the most from the improved low frequency sensitivity of ET-D. We also showed that at least for a narrower parameter space it would be possible to put stringent bounds on the first two spin-induced multipole moments (quadrupolar and octupolar) simultaneously to assess the nature of the involved compact binary (see Sec. IV B above). This also means one would be able to constrain four multipole moments of the compact binary system facilitating a thorough probe of their binary black hole nature. Finally, the possibility of bounding the leading spin-induced moment for each binary component was explored in Sec. IV C. Results of Fig. 8 suggest a set of possible binary configurations for which at least the nature of the heavier component can be confirmed.

Due to the dependencies of the bounds on component spins, mass ratios, and masses, it is somewhat difficult to predict the magnitude of the constraints these measurements will place on the parameter space of BH mimickers. For example, considering boson star models of Ryan [70], the theoretically allowed lower limit on the quadrupole parameter is $\sim 10$ (see Fig. 4 of [70]) and lower limit on the octupole parameter is $\sim 20$ (Fig. 5 of [70]). These values do depend on parameters such as the mass of the boson which constitute the boson stars. Figure 3 above shows that, unless component spin values are less than 0.1 , the expected bounds on $\kappa_{s}$ will rule out boson star models 
which predict $\kappa_{s} \sim 10$. Similarly, as can be seen from Fig. 7, detecting moderate to highly spinning systems $(\chi \sim 0.4-0.9)$ can help you rule out $\lambda_{s} \sim 20$. However, it has to be borne in mind that the constraints from individual events can rule out a BH mimicker association only for that system and not a generic constrain on the parameter space of $\mathrm{BH}$ mimickers. The constraints on gravastars, however, are likely to be weaker as the spin-induced quadrupole moment parameter of this class of objects spans a small range of values around the $\mathrm{BH}$ value $(\sim-0.17-1.8)$ as can be seen from Fig. 7 of [90].
Acknowledgment: The authors thank M. Saleem for useful discussions. We thank Anuradha Gupta for useful comments on the manuscript. K. G. A. and N. V. K. were partially supported by a grant from Infosys foundation. K. G. A. acknowledges the Indo-US Science and Technology Forum through the Indo-US Centre for the Exploration of Extreme Gravity, grant IUSSTF/JC-029/2016. K. G. A. also acknowledges partial support by the Grant No. EMR/2016/005594 of SERB. This document has LIGO preprint number P1800325.
[1] B. P. Abbott et al. (Virgo, LIGO Scientific), Phys. Rev. Lett. 116, 061102 (2016), 1602.03837.

[2] B. P. Abbott et al. (Virgo, LIGO Scientific), Phys. Rev. Lett. 116, 241103 (2016), 1606.04855.

[3] B. P. Abbott et al. (VIRGO, LIGO Scientific), Phys. Rev. Lett. 118, 221101 (2017), [Erratum: Phys. Rev. Lett.121,no.12,129901(2018)], 1706.01812.

[4] B. P. Abbott et al. (Virgo, LIGO Scientific), Astrophys. J. 851, L35 (2017), 1711.05578.

[5] B. P. Abbott et al. (Virgo, LIGO Scientific), Phys. Rev. Lett. 119, 141101 (2017), 1709.09660.

[6] B. P. Abbott et al. (LIGO Scientific, Virgo) (2018), 1811.12907.

[7] B. P. Abbott et al. (LIGO Scientific, Virgo) (2018), 1811.12940.

[8] J. Meidam, M. Agathos, C. Van Den Broeck, J. Veitch, and B. S. Sathyaprakash, Phys. Rev. D90, 064009 (2014), 1406.3201.

[9] N. Yunes and X. Siemens, Living Rev. Rel. 16, 9 (2013), 1304.3473 .

[10] K. G. Arun, B. R. Iyer, M. S. S. Qusailah, and B. S. Sathyaprakash, Class. Quant. Grav. 23, L37 (2006), grqc/0604018.

[11] K. G. Arun, B. R. Iyer, M. S. S. Qusailah, and B. S. Sathyaprakash, Phys. Rev. D74, 024006 (2006), gr-qc/0604067.

[12] E. Berti, K. Yagi, and N. Yunes, Gen. Rel. Grav. 50, 46 (2018), 1801.03208.

[13] E. Berti, K. Yagi, H. Yang, and N. Yunes, Gen. Rel. Grav. 50, 49 (2018), 1801.03587.

[14] C. M. Will, Astrophys. J. 214, 826 (1977).

[15] M. Agathos, W. Del Pozzo, T. G. F. Li, C. Van Den Broeck, J. Veitch, and S. Vitale, Phys. Rev. D89, 082001 (2014), 1311.0420 .

[16] N. Yunes and F. Pretorius, Phys. Rev. D80, 122003 (2009), 0909.3328.

[17] C. M. Will, Phys. Rev. D57, 2061 (1998), gr-qc/9709011.

[18] A. Samajdar and K. G. Arun, Phys. Rev. D96, 104027 (2017), 1708.00671 .

[19] A. Ghosh, N. K. Johnson-Mcdaniel, A. Ghosh, C. K. Mishra, P. Ajith, W. Del Pozzo, C. P. L. Berry, A. B. Nielsen, and L. London (2017), 1704.06784.

[20] B. P. Abbott et al. (Virgo, LIGO Scientific), Phys. Rev. Lett. 116, 221101 (2016), 1602.03841.

[21] B. P. Abbott et al. (Virgo, LIGO Scientific), Phys. Rev. X6, 041015 (2016), 1606.04856.

[22] L. Blanchet, Living Reviews in Relativity 17, 2 (2014), 1310.1528 .

[23] F. Pretorius (2007), 0710.1338.

[24] M. Sasaki and H. Tagoshi, Living Rev. Rel. 6, 6 (2003), grqc/0306120.

[25] G. F. Giudice, M. McCullough, and A. Urbano, jcap 10, 001 (2016), 1605.01209.

[26] B. Sathyaprakash et al., in Proceedings, 46th Rencontres de Moriond on Gravitational Waves and Experimental Gravity:
La Thuile, Italy, March 20-27, 2011 (2011), pp. 127-136, 1108.1423.

[27] T. Regimbau et al., Phys. Rev. D86, 122001 (2012), 1201.3563.

[28] S. Hild et al., Class. Quant. Grav. 28, 094013 (2011), 1012.0908.

[29] S. Hild, S. Chelkowski, and A. Freise (2008), 0810.0604.

[30] P. O. Mazur and E. Mottola, Proceedings of the National Academy of Science 101, 9545 (2004), gr-qc/0407075.

[31] S. L. Liebling and C. Palenzuela, Living Reviews in Relativity 15, 6 (2012), 1202.5809.

[32] A. Almheiri, D. Marolf, J. Polchinski, and J. Sully, JHEP 02, 062 (2013), 1207.3123.

[33] R. O. Hansen, Journal of Mathematical Physics 15, 46 (1974).

[34] B. Carter, Physical Review Letters 26, 331 (1971).

[35] N. Gürlebeck, Phys. Rev. Lett. 114, 151102 (2015), 1503.03240.

[36] F. D. Ryan, Phys. Rev. D52, 5707 (1995).

[37] R. Geroch, Journal of Mathematical Physics 11, 2580 (1970).

[38] R. Geroch, Journal of Mathematical Physics 11, 1955 (1970).

[39] T. Binnington and E. Poisson, Phys. Rev. D 80, 084018 (2009).

[40] N. Gürlebeck, Physical Review Letters 114, 151102 (2015), 1503.03240.

[41] É. É. Flanagan and T. Hinderer, Phys. Rev. D 77, 021502 (2008), 0709.1915.

[42] J. Vines, É. É. Flanagan, and T. Hinderer, Phys. Rev. D83, 084051 (2011), 1101.1673.

[43] V. Cardoso, E. Franzin, A. Maselli, P. Pani, and G. Raposo (2017), 1701.01116.

[44] N. Sennett, T. Hinderer, J. Steinhoff, A. Buonanno, and S. Ossokine, Phys. Rev. D96, 024002 (2017), 1704.08651.

[45] N. K. Johnson-Mcdaniel, A. Mukherjee, R. Kashyap, P. Ajith, W. Del Pozzo, and S. Vitale (2018), 1804.08026.

[46] C. V. Vishveshwara, nature 227, 936 (1970).

[47] O. Dreyer, B. Kelly, B. Krishnan, L. S. Finn, D. Garrison, and R. Lopez-Aleman, Class. Quantum Grav. 21, 787 (2004), grqc/0309007.

[48] E. Berti, V. Cardoso, and A. O. Starinets, Class. Quant. Grav. 26, 163001 (2009), 0905.2975.

[49] C. F. B. Macedo, V. Cardoso, L. C. B. Crispino, and P. Pani, Phys. Rev. D93, 064053 (2016), 1603.02095.

[50] E. Berti and V. Cardoso, Int. J. Mod. Phys. D15, 2209 (2006), gr-qc/0605101.

[51] C. F. B. Macedo, P. Pani, V. Cardoso, and L. C. B. Crispino, Phys. Rev. D88, 064046 (2013), 1307.4812.

[52] C. B. M. H. Chirenti and L. Rezzolla, Classical and Quantum Gravity 24, 4191 (2007).

[53] P. Pani, E. Berti, V. Cardoso, Y. Chen, and R. Norte, Phys. Rev. D 80, 124047 (2009), 0909.0287.

[54] C. B. M. H. Chirenti and L. Rezzolla, Class. Quant. Grav. 24, 4191 (2007), 0706.1513.

[55] J. B. Hartle, Phys. Rev. D8, 1010 (1973).

[56] K. Chatziioannou, E. Poisson, and N. Yunes, Phys. Rev. D87, 044022 (2013), 1211.1686. 
[57] K. Chatziioannou, E. Poisson, and N. Yunes, Phys. Rev. D94, 084043 (2016), 1608.02899.

[58] A. Maselli, P. Pani, V. Cardoso, T. Abdelsalhin, L. Gualtieri, and V. Ferrari (2017), 1703.10612.

[59] F. D. Ryan, Phys. Rev. D56, 1845 (1997).

[60] C. L. Rodriguez, I. Mandel, and J. R. Gair, Phys. Rev. D85, 062002 (2012), 1112.1404

[61] D. A. Brown, H. Fang, J. R. Gair, C. Li, G. Lovelace, I. Mandel, and K. S. Thorne, Phys. Rev. Lett. 99, 201102 (2007), grqc/0612060.

[62] N. A. Collins and S. A. Hughes, Phys. Rev. D 69, 124022 (2004), gr-qc/0402063.

[63] K. Glampedakis and S. Babak, Classical and Quantum Gravity 23, 4167 (2006), gr-qc/0510057.

[64] A. Ghosh, N. K. Johnson-McDaniel, A. Ghosh, C. Kant Mishra, P. Ajith, W. Del Pozzo, C. P. L. Berry, A. B. Nielsen, and L. London, Classical and Quantum Gravity 35, 014002 (2018), 1704.06784.

[65] J. Healy, C. O. Lousto, and Y. Zlochower, Phys. Rev. D90, 104004 (2014), 1406.7295.

[66] N. V. Krishnendu, K. G. Arun, and C. K. Mishra, Phys. Rev. Lett. 119, 091101 (2017), 1701.06318.

[67] W. G. Laarakkers and E. Poisson, Astrophys. J. 512, 282 (1999), gr-qc/9709033.

[68] G. Pappas and T. A. Apostolatos (2012), 1211.6299.

[69] G. Pappas and T. A. Apostolatos, Physical Review Letters 108, 231104 (2012), 1201.6067.

[70] F. D. Ryan, Phys. Rev. D 55, 6081 (1997).

[71] S. Dwyer, D. Sigg, S. W. Ballmer, L. Barsotti, N. Mavalvala, and M. Evans, Phys. Rev. D 91, 082001 (2015), 1410.0612.

[72] L. Blanchet, Living Rev. Rel. 17, 2 (2014), 1310.1528.

[73] L. Blanchet, T. Damour, G. Esposito-Farèse, and B. R. Iyer, Phys. Rev. Lett. 93, 091101 (2004), gr-qc/0406012.

[74] L. Blanchet, G. Faye, B. R. Iyer, and B. Joguet, Phys. Rev. D 65, 061501(R) (2002), Erratum-ibid 71, 129902(E) (2005), gr-qc/0105099.

[75] L. Blanchet, T. Damour, B. R. Iyer, C. M. Will, and A. G. Wiseman, Phys. Rev. Lett. 74, 3515 (1995), gr-qc/9501027.

[76] S. Marsat, A. Bohé, G. Faye, and L. Blanchet, Class. Quant. Grav. 30, 055007 (2013), 1210.4143.

[77] A. Bohé, S. Marsat, G. Faye, and L. Blanchet, Class. Quant.
Grav. 30, 075017 (2013), 1212.5520.

[78] A. Bohé, S. Marsat, and L. Blanchet, Class. Quant. Grav. 30, 135009 (2013), 1303.7412.

[79] S. Marsat, A. Bohé, L. Blanchet, and A. Buonanno, Class. Quant. Grav. 31, 025023 (2014), 1307.6793.

[80] A. Bohé, G. Faye, S. Marsat, and E. K. Porter, Class. Quant. Grav. 32, 195010 (2015), 1501.01529.

[81] S. Marsat, Class. Quant. Grav. 32, 085008 (2015), 1411.4118.

[82] K. G. Arun, A. Buonanno, G. Faye, and E. Ochsner, Phys. Rev. D79, 104023 (2009), [Erratum: Phys. Rev.D84,049901(2011)], 0810.5336 .

[83] L. E. Kidder, Phys. Rev. D52, 821 (1995), gr-qc/9506022.

[84] C. M. Will and A. G. Wiseman, Phys. Rev. D54, 4813 (1996), gr-qc/9608012.

[85] A. Buonanno, G. Faye, and T. Hinderer, Phys. Rev. D87, 044009 (2013), 1209.6349.

[86] C. K. Mishra, A. Kela, K. G. Arun, and G. Faye (2016), 1601.05588 .

[87] E. Poisson, Phys. Rev. D57, 5287 (1998), gr-qc/9709032.

[88] P. O. Mazur and E. Mottola, Proc. Nat. Acad. Sci. 101, 9545 (2004), gr-qc/0407075.

[89] N. Uchikata, S. Yoshida, and P. Pani, Phys. Rev. D94, 064015 (2016), 1607.03593.

[90] N. Uchikata and S. Yoshida, Class. Quant. Grav. 33, 025005 (2016), 1506.06485.

[91] C. Cutler and E. E. Flanagan, Phys. Rev. D49, 2658 (1994), gr-qc/9402014.

[92] C. Rao, Bullet. Calcutta Math. Soc 37, 81 (1945).

[93] H. Cramer, Mathematical methods in statistics (Pergamon Press, Princeton University Press, NJ, U.S.A., 1946).

[94] S. Husa, S. Khan, M. Hannam, M. Pürrer, F. Ohme, X. Jiménez Forteza, and A. Bohé, Phys. Rev. D93, 044006 (2016), 1508.07250.

[95] M. Favata, K. G. Arun, C. Kim, J. Kim, and H. W.Lee (In preparation)

[96] B. P. Abbott et al. (LIGO Scientific) (2016), 1607.08697.

[97] S. Hild, M. Abernathy, F. Acernese, P. Amaro-Seoane, N. Andersson, K. Arun, F. Barone, B. Barr, M. Barsuglia, M. Beker, et al., Classical and Quantum Gravity 28, 094013 (2011). 